\title{
Neuromimetic encoding/decoding of spatiotemporal spiking signals from an artificial touch sensor
}

\author{
Luca Leonardo Bologna*, Romain Brasselet*, Marco Maggiali, Angelo Arleo
}

\begin{abstract}
A framework to discriminate tactile stimuli delivered to an artificial touch sensor is presented.

Following a neuromimetic approach, we encode the signals from a 24-capacitive sensor fingertip into spiking activity through a network of leaky integrate-and-fire neurons. The activity resulting from the stimulation of the touch sensor through Braille-like dot patterns is then analysed by means of a newly defined Information measure which explicitly takes into consideration the metrics of the spike train space.

Results show that an optimal discrimination of the entire set of 26 stimuli (i.e. $100 \%$ correct classification) is reached early after the stimulus onset. Interestingly, the method proves to be effective with both statically and dynamically delivered stimulation which are hard to decode because of the similarity between encoded firing activity given to the proximity of the patterns presented.

The decoding analysis allowed us to corroborate the working hypothesis that human tactile discrimination relies on optimal encoding/decoding processes already at the level of the primary stage neurons in the somatosensory pathway.
\end{abstract}

\section{INTRODUCTION}

Fast and reliable tactile discrimination plays a paramount role in human behaviour in order to guarantee rapid response and adaptation to stimuli delivered to the fingertips. Even simple object manipulation requires the ability to identify the object's properties and perform optimal action selection based on closed-loop control policies. The same holds for humanoid robotics applications in which human-like haptic tasks must rely on high stability, precision and adaptability of the system. More specifically, at the early stages of the ascending pathway, there must be a faithful encoding of the tactile stimulations into populations of spike trains, so that the central nervous system can actually decode the signals and discriminate the stimulations.

Here we study how to decode spiking activity obtained by encoding analogue data from an artificial touch sensor. We show that, after stimulating the sensor with Braille-like dot

\footnotetext{
* Both authors contributed equally to this work.

Luca Leonardo Bologna is with the Adaptive NeuroComputation Group, Unit of Neurobiology of Adaptive Processes, CNRS - University Pierre\&Marie Curie, 75005 Paris, France (phone: +33 (0)1 442721 33; email: luca.bologna@upmc.fr).

Romain Brasselet is with the Adaptive NeuroComputation Group, Unit of Neurobiology of Adaptive Processes, CNRS - University Pierre\&Marie Curie, 75005 Paris, France (phone: +33 (0)1 442721 33; email: rbrassel@ snv.jussieu.fr).

Marco Maggiali is with the Department of Robotics, Brain and Cognitive Sciences, Italian Institute of Technology, Genoa, Italy (phone: +33 3316000360; email: marco.maggiali@iit.it).

Angelo Arleo is with the Adaptive NeuroComputation Group, Unit of Neurobiology of Adaptive Processes, CNRS - University Pierre\&Marie Curie, 75005 Paris, France (phone: +33 (0)1 442727 80; email: angelo.arleo@upmc.fr)
}

patterns both statically and dynamically (i.e. by rubbing the pattern over the finger), it is possible to quickly recover the stimulus on both modality. Drawing inspiration from a study of human haptic microneurography spiking data, we use a new metrical information coupled with the Victor-Purpura distance [13] to estimate the amount of information that can be transmitted without burst of uncertainty on the response to a single stimulus.

\section{Methods}

\section{A. The encoding/decoding scheme}

In Fig. 1 we show the entire encoding/decoding scheme. After stimulating the artificial fingertip with scaled Braillecharacter probes the analogue data were converted into spiking activity through a network of modified leaky integrateand-fire neurons (LIF). The obtained activity was then analysed through an Information Theory based approach (cf. section II-D) in order to classify the response of the fingertip to both static and dynamical stimuli.

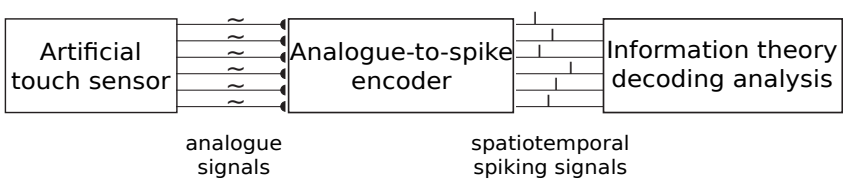

Fig. 1. The entire encoding/decoding process. After the conversion of the finger output signals into spike trains, the information theoretical method is applied in order to discriminate between different stimuli.

\section{B. The artificial fingertip}

The skin prototype was developed at the Italian Institute of Technology (IIT, Genoa, Italy) as a small-scale improvement of a previously presented artificial skin [3]. The entire artificial finger has a sensitive surface of approximately $18 \mathrm{~mm} x$ $23 \mathrm{~mm}$. It consists of 24 capacitive square sensors disposed according to a rectangular grid layout. The dimension of each sensor is approximately $3 \mathrm{~mm}$ and the inter-centre distance is $4 \mathrm{~mm}$ (Fig. 2).

The array is covered by a $2.5 \mathrm{~mm}$ thick neoprene layer in order to modulate the pressure exerted over the sensors. The higher the indentation of a conductive material on the neoprene is, the stronger the response of the sensors. The response strength of each sensor ranges between 0 and 189 fF. 

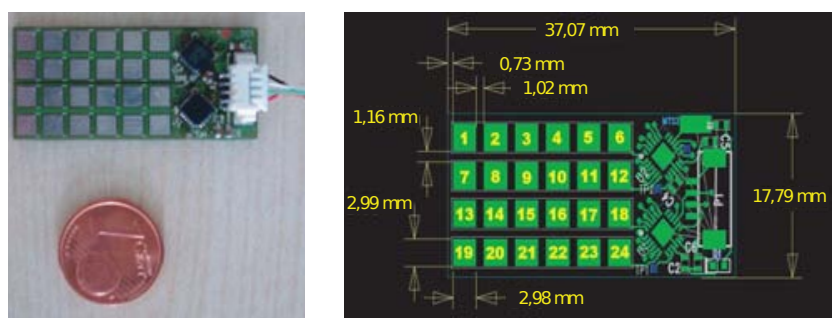

Fig. 2. Left: The artificial fingertip consisting of an array of 24 capacitive sensors. Right: Design of the fingertip.

\section{The analog-to-spike conversion method}

A network of 24 modified leaky integrate-and-fire neurons [5] was modeled to convert the analogue signals provided by the artificial skin after stimulation into spatiotemporal spike patterns (Fig. 3).

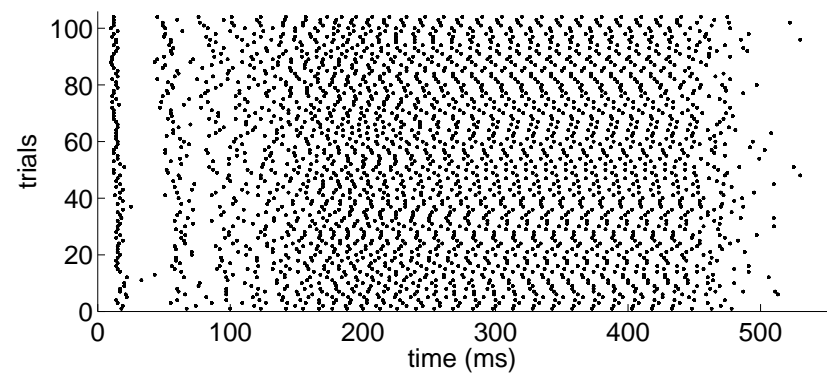

Fig. 3. Spike trains obtained through the encoding process of analogue signals. The response of 8 sensors to a single probe stimulation repeated 13 times is reported.

1) The spiking neural network: Let $V_{\text {leak }}, V_{\text {thr }}, V_{\text {reset }}$ and $I(t)$ denote the resting membrane potential, the firing threshold, the reset potential, and the total synaptic input of a neuron, respectively. The dynamics of the membrane potential $V(t)$ was defined according to:

$$
C \cdot \frac{d V(t)}{d t}=-g \cdot\left(V(t)-V_{\text {leak }}\right)-I(t)
$$

with $C$ and $g$ being the membrane capacitance and leak conductance, respectively. We took $V_{\text {leak }}=-70 \mathrm{mV}, V_{t h r}=$ $-50 \mathrm{mV}$, and $V_{\text {reset }}=-100 \mathrm{mV}$ for all neurons of the model. The membrane capacitance $C$ was taken equal to $0.5 \mathrm{nF}$, and the conductance $g$ equal to $25 \mathrm{nS}$. Thus, the membrane time constant was $\tau=C / g=20 \mathrm{~ms}$. Whenever the membrane potential $V(t)$ reached the threshold $V_{t h r}$ the neuron emitted one spike. Then, its membrane potential was reset to $V_{\text {reset }}$ and the dynamics of $V(t)$ was frozen during a refractory period $\Delta t_{r e f}=2 \mathrm{~ms}$. A basic "threshold fatigue" [4] was also implemented in order to model the phenomenon of "habituation". It consisted in increasing the threshold $V_{t h r}$ of a value $A_{t h r}$ each time the neuron spiked, making it harder for the neuron to spike again (i.e. preventing the neuron from responding highly tonically even in the presence of strong inputs). In the absence of spikes, the threshold decreased exponentially back to its resting value $V_{\text {restThr }}$ :

$$
\frac{d V_{t h r}(t)}{d t}=-\frac{V_{t h r}(t)-V_{r e s t T h r}}{\tau_{t h r}}
$$

The parameter values we used were $\tau_{t h r}=100 \mathrm{~ms}$, $V_{\text {restThr }}=-50.0 \mathrm{mV}$ and $A_{t h r}=50.0 \mathrm{mV}$. Eqs. 1 and 2 were integrated using Runge-Kutta 2 and a timestep of 1 ms.

2) The receptive fields: Each neuron $i$ received a driving input based on its receptive field function, which was used to sample the data space similar to a kernel basis function (Fig. 4a). The input $I(t)$ was then simply taken as:

$$
I(t)=\sum_{j} w_{i j} \cdot d_{j}
$$

with $w_{i j}$ representing the weight of the "connection" from a capacitive sensor $j$ to the neuron, and $d_{j}$ being the analogue value of the fingertip sensor. Two types of receptive fields were implemented and tested: the Gaussian function and the Heaviside kernel (Fig. 4b and 4c). The weights $w_{j}$ were normalised to obtain a global maximum weight defined by a constant $W=\sum_{j} w_{j}$. Given the properties of the artificial fingertip and the dimension and property of each sensors (cf. section III-A) the results we present were obtained using a Gaussian kernel with radius $\ll 1$ approximately equivalent to an Heaviside kernel with radius < 1 (Fig. 4).

\section{Information-theoretic measure}

Mutual information ( $M I)$ [9], [11] measures the interdependence of two random variables and it was defined by Shannon as follows:

$$
M I(s ; r)=\sum_{s} \sum_{r} p(r, s) \log \frac{p(r, s)}{p(r) p(s)}
$$

where $r$ denotes the response to a given stimulus $s$. In the classical Shannon definition, the terms $p(s)$ and $p(r)$ are the marginal probabilities of stimulus and response, respectively, $p(r, s)$ is their joint probability, and $p(r \mid s)$ indicates the conditional probability.

In order to decode the neural activity and discriminate between different stimuli, we applied a novel information theoretical measure $M I(R ; S)^{*}$ following two new definitions for the marginal and conditional entropy defined as $H(R)^{*}$ and $H^{*}(R \mid S)$ respectively. Such an information measure, which has been proven to be suitable to decode the responses of real mechanoreceptors obtained via microneurography recordings in humans [2], was derived analytically. It takes into account the metrical properties of the spike train space [10], [12] in order to circumvent the problem related to binning procedures encountered when applying information theoretic analysis [8], [6].

1) Victor-Purpura distance: The metrics we chose is the Victor-Purpura spike train metrics [13] which defines the distance between two spike trains as the minimum cost of the operations needed to transform one spike train into the other. Briefly, the three allowed operations and their respective costs are: 


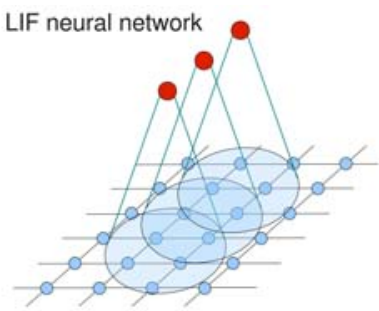

(b)
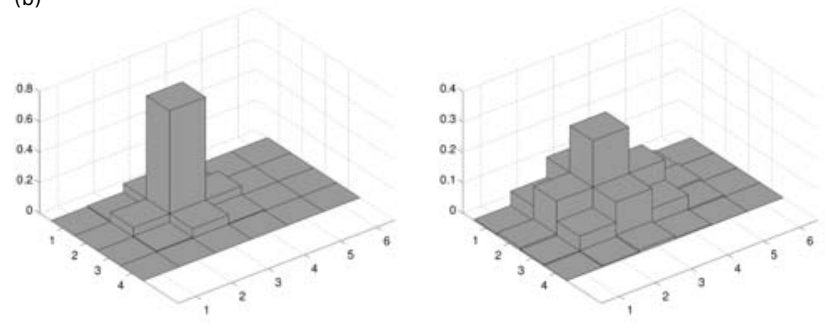

(c)
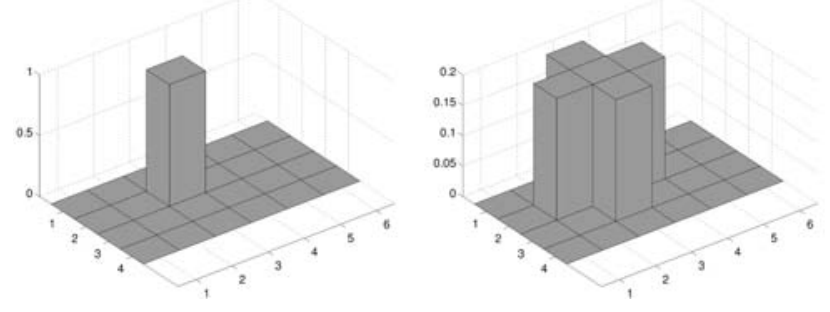

Fig. 4. (a) The input of each LIF neuron was computed on the basis of its receptive field. (b) Examples of Gaussian receptive fields. Left: standard deviation $=0.6$. Right: standard deviation $=1.2$. (c) Examples of Heaviside receptive fields. Left: radius $<1$ (Dirac equivalent). Right: radius $=1$.

- the insertion of a single spike at a fixed cost of 1 ,

- the deletion of a single spike at a fixed cost of 1 ,

- the displacement in time of one spike for a cost that grows linearly with the temporal shift. This cost depends on the parameter $C_{V P}$ which has to be appropriately chosen. According to the given definition, moving a spike by an interval $\Delta t$ will cost $C_{V P} \cdot \Delta t$.

In order to extend this metrics to a neural population we simply summed individual distances over each neuron of the network to obtain the population distance $D_{V P}\left(r, r^{\prime}\right)$. We then used such a distance to compute the degree of similarity between responses elicited by the same stimulus (i.e. intrastimulus distance) and responses elicited by different stimuli (i.e. interstimulus distance).

2) Metrical Information: The novel measure we used for the marginal entropy, namely the uncertainty on the response to the whole stimulus set, is formalised as follows:

$$
H^{*}(R)=-\sum_{r \in R} \frac{1}{|R|} \log \sum_{r^{\prime} \in R} \frac{<r \mid r^{\prime}>}{|R|}
$$

where $<r \mid r^{\prime}>$ is a similarity measure between two responses $r$ and $r^{\prime}$ (see below). Then, the metrical conditional entropy, indicating the uncertainty on the response to a single stimulus, is defined as:

$$
\begin{array}{r}
H^{*}(R \mid S)=\sum_{s \in S} p(s) H^{*}(R \mid s)= \\
-\sum_{s \in S} p(s) \sum_{r \in R_{s}} \frac{1}{\left|R_{s}\right|} \log \sum_{r^{\prime} \in R_{s}} \frac{<r \mid r^{\prime}>}{\left|R_{s}\right|}
\end{array}
$$

Finally, the metrical information is defined, following the Shannon's definition, as the difference between the marginal and conditional entropies:

$$
I^{*}(R ; S)=H^{*}(R)-H^{*}(R \mid S)
$$

The similarity measure $\left\langle r \mid r^{\prime}\right\rangle$ is defined as a function of the VP distance $D_{V P}\left(r, r^{\prime}\right)$ between two population responses $r$ and $r^{\prime}$. More specifically, we defined $\left\langle r \mid r^{\prime}\right\rangle$ as follows:

$$
<r \mid r^{\prime}>=1 \Longleftrightarrow D_{V P}\left(r, r^{\prime}\right)<D_{\text {critic }}
$$

where the critical distance $D_{\text {critic }}$ is a free parameter, interdependent to $C_{V P}$, that needs to be determined properly. More specifially, in order to determine the optimal values for $D_{\text {critic }}$ and $C_{V P}$ we computed the minimum and maximum interstimuli and intrastimuli distances. According to the novel information theoretical measures, a perfect discrimination between different stimuli, which corresponds to maximal $I^{*}(R ; S)$ and zero $H^{*}(R \mid S)$ occurs when the maximum intrastimuli distance becomes smaller than the minimal intrerstimulus distance. This means that all the responses to a given stimulus should lie in a region of space whose size is smaller than the distance to the closest response to another stimulus.

\section{E. Experimental protocols}

Four different experimental protocols were applied to the sensor array in order to both characterise its properties and collect a data benchmark to which apply the discrimination method (cf. section II-E). The $1^{\text {st }}$ experimental session aimed at characterising the response of the device with respect to the strength and position of the stimulus so to precisely define the receptive field of each sensor. A single $4 \mathrm{~mm}$-diameter cylindrical probe, mounted on a load cell, was used to indent the neoprene. The touch sensor was stimulated at 6161 different positions over the entire array surface according to a regular grid layout (i.e. $0.2 \mathrm{~mm}$ step). During the $2^{\text {nd }}$ session, the same probe was rubbed over the entire finger at constant force amplitude. The protocol was repeated at two different sliding velocities in order to test the response of the finger. The $3^{r d}$ and the $4^{\text {th }}$ sessions aimed at emulating Braille-like reading tasks. In the $3^{r d}$ session a set of 26 probes reproducing a scaled version $(1: 3)$ of the Braille alphabet was used as stimulation probe. All the patterns where indented three times at two different alignments with respect to the sensor array (156 static presentations). During the $4^{t h}$ session, the set of 26 single characters were rubbed over the fingertip three times at two different velocities and two different alignments (444 dynamical pattern presentations). 


\section{RESULTS}

\section{A. Fingertip characterization}

A characterization of the basic properties of the fingertip responses is reported in Fig. 5. The results refer to data collected when the probe was entirely indented into the neoprene. Each of the 24 sensors shows a high signal-tonoise ratio $(50 \mathrm{~dB})$ and a Gaussian-shaped receptive field with amplitude $200 \mathrm{fF} \pm 3.3$ std and width $2.5 \mathrm{~mm} \pm$ $0.044 \mathrm{std}$. We also report the fitted responses of 4 adjacent sensors positioned at the center of the finger. The fitting procedure clearly shows the stability of the device whose sensors respond in a similar way regardless of the position they have in the finger layout. It is also possible to observe the degree of overlapping between sensors' receptive fields. As a supplementary analysis, the fitting procedure was also perfomed by reading out the analogue signals at different indentation levels of the probe. The same gaussian shape of the response with equal receptive field widths and amplitude proportional to the indentation of the probe was observed (data not shown).
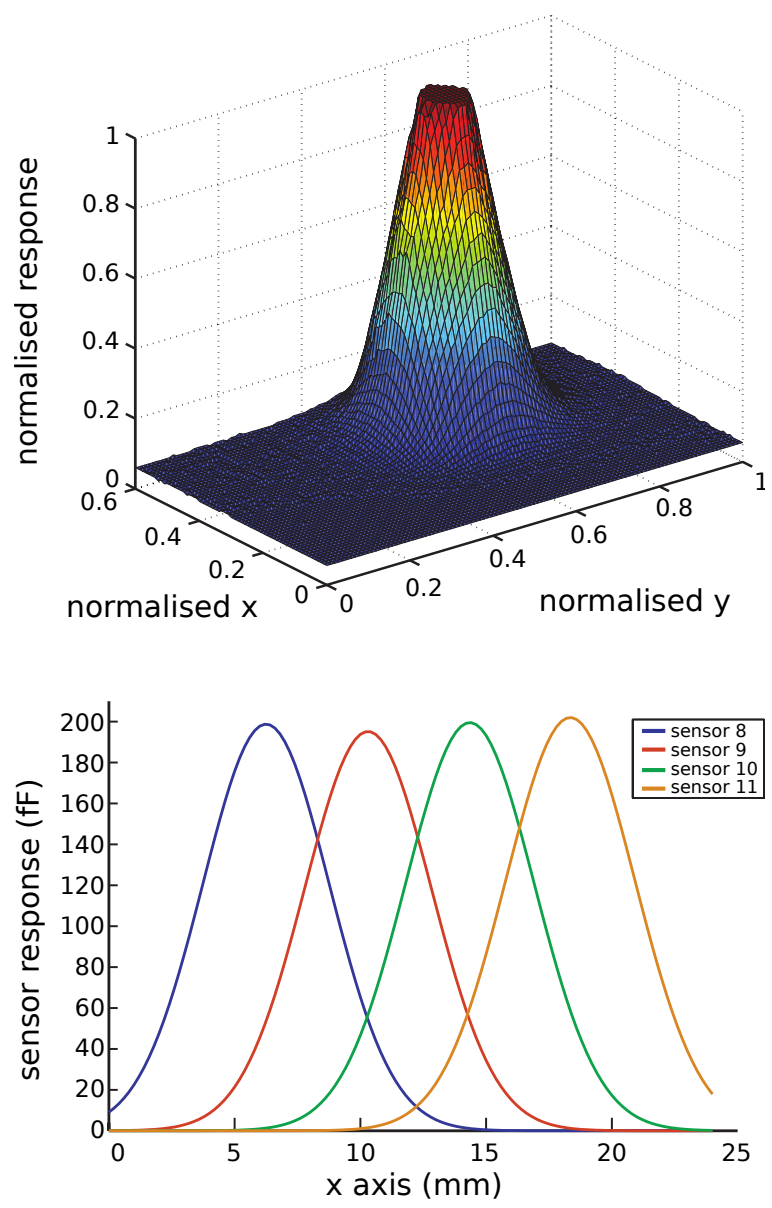

Fig. 5. Top: Normalised receptive field of a single sensor obtained by sampling its response at 6161 different positions over the entire array (axis dimensions were normalised). Bottom: Gaussian fitting of the response of 4 sensors along a line.

\section{B. Encoding of analogue data into spiking activity}

We report in Fig. 6 two examples of spiking activity obtained after encoding the analogue data obtained upon stimulation of the fingertip with a scaled Braille "F" character. One can clearly observe that firing rates change according to the strength of the stimulation delivered to the sensors. In fact, the closer is the probe to a sensor the stronger is its analogue response driving to a higher firing activity of the neurons encoding its signal. Moreover, given the dynamics governing the LIF neurons, a longer stimulation induces a constantly substained firing activity as shown in the raster plot obtained from the static stimulation. Here, the three neurons encoding the activity of the channels which were closer to the probe show a bursting activity unobserved from the others. Furthermore, it can be noticed how in the actvity encoded upon dynamical stimulation the firing patterns of the most solicited neurons change in time as the probe is rubbed. Such a change reproduce a topological mapping which links the activity of the neurons to the area of stimulation. A similar behaviour has been observed for a particular type of human fingertip mechanoreceptors (i.e. SAI) measured in experiments involving Braille character scanning [7]. In order to apply the information-theory method, for both the static and dynamic protocols we generated 100 activity patterns for each character by taking the activity encoded from one of the performed experiments and adding a $3 \mathrm{~mm}$ jitter to each spike from a uniform distribution.

\section{Statically delivered stimuli data}

In Fig. 7 we report the results of the discrimination of all Braille alphabet letters statically delivered to the fingertip. Only the first spike waves were taken into consideration for the decoding analysis in order to demonstrate the high information content in the timing of the first spikes as reported in previous works [1]. Due to the use of the novel metrical information measure (cf. section II-D), a perfect discrimination is possible very early with respect to the stimulus onset (i.e. after $120 \mathrm{~ms}$ ). This result extend to artificial data previous observations in human microneurography data in which the stimulus source could be perfectly discriminated in few tens of milliseconds [2], [1]. Such an outcome is particularly encouraging for robotic application where a fast response to external stimuli is required. Furthermore, a spike based discrimination method allows to exploit the complexity of the neural code by taking advantage of the variety of both the spatial and temporal dynamics of firing patterns in populations of neurons.

\section{Dynamically delivered stimuli data}

In Fig. 8 the results concerning the discrimination of all Braille alphabet letters dynamically delivered are reported. Given the different nature of the experiments, the need is to analyse the firing pattern over stimulation time as it changes together with the position of the probe. Hence, differently from the static protocol, the entire spike train of each neuron was taken into consideration for the decoding analysis and 


\section{LIF neuron response to Braille character :} $(F)$
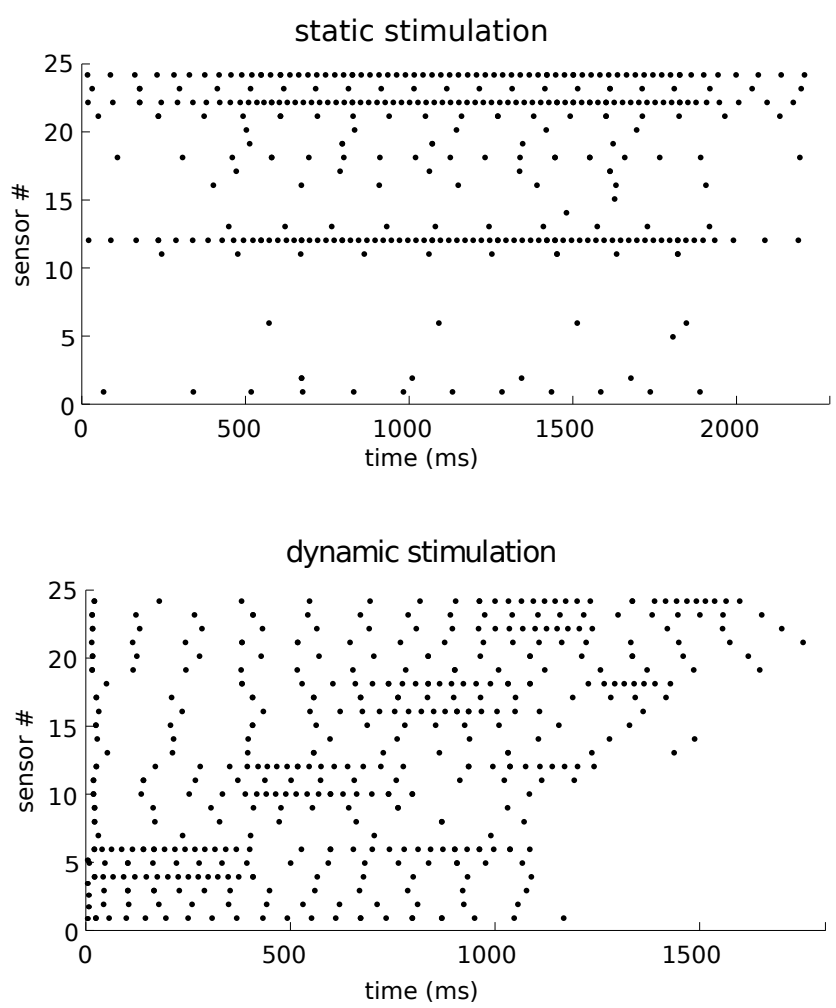

Fig. 6. Spiking activity of the LIF neural network after enconding the analogue data of the fingertip in response to stimulation through the " $\mathrm{F}$ " Braille character. Top: raster obtained after static stimulation encoding. One can observe that neurons 12,22 and 24 , encoding the activiy of sensors named 12, 22, and 24 in the touch sensor layout (Fig. 2) show high firing rates because of the proximity of such sensors with the stimulation probe. Differently, the other neurons show an extremely low firing rate (if any) because they take into account capacitive signals mainly due to noisy effect given to the indentation of the probe into the fingerip. Bottom: raster obtained after dynamical stimulation encoding. As for static stimulation few neurons are more solicited than the others but, in this case, the firing patterns change in time clearly following the movement of the probe while it is rubbed over the finger. Also in this case noisy effect are observed, which are higher than in the static stimulaion because of the strongest solicitation all the sensors undergo during the movement of the probe. Nonetheless, one can clearly distinguish the high activity of the neurons whose solicitation is given by a wider indentation of the probe with respect to the ones undergoing only noisy effects.

not only the firts spike wave. Also in this case, as shown for the static stimulus, a perfect discrimination is possible once the minimum interstimuli has overcome the maximum intrastimuli distance (cf. section II-D). Again, the latter condition is verified very early after the stimulus onset at around $500 \mathrm{~ms}$ when maximum information and minimum conditional entropy occur. It should be remarked that the method we used proves to be particularly powerful even in the presence of long spike trains. In fact, as a higher number of spike is considered the computed interstimuli and intrastimuli distances tends to create wider clusters of responses whose overlapping tends to be more probable. Nonetheless, the choice of an appropriate cost $C_{V P}$ and
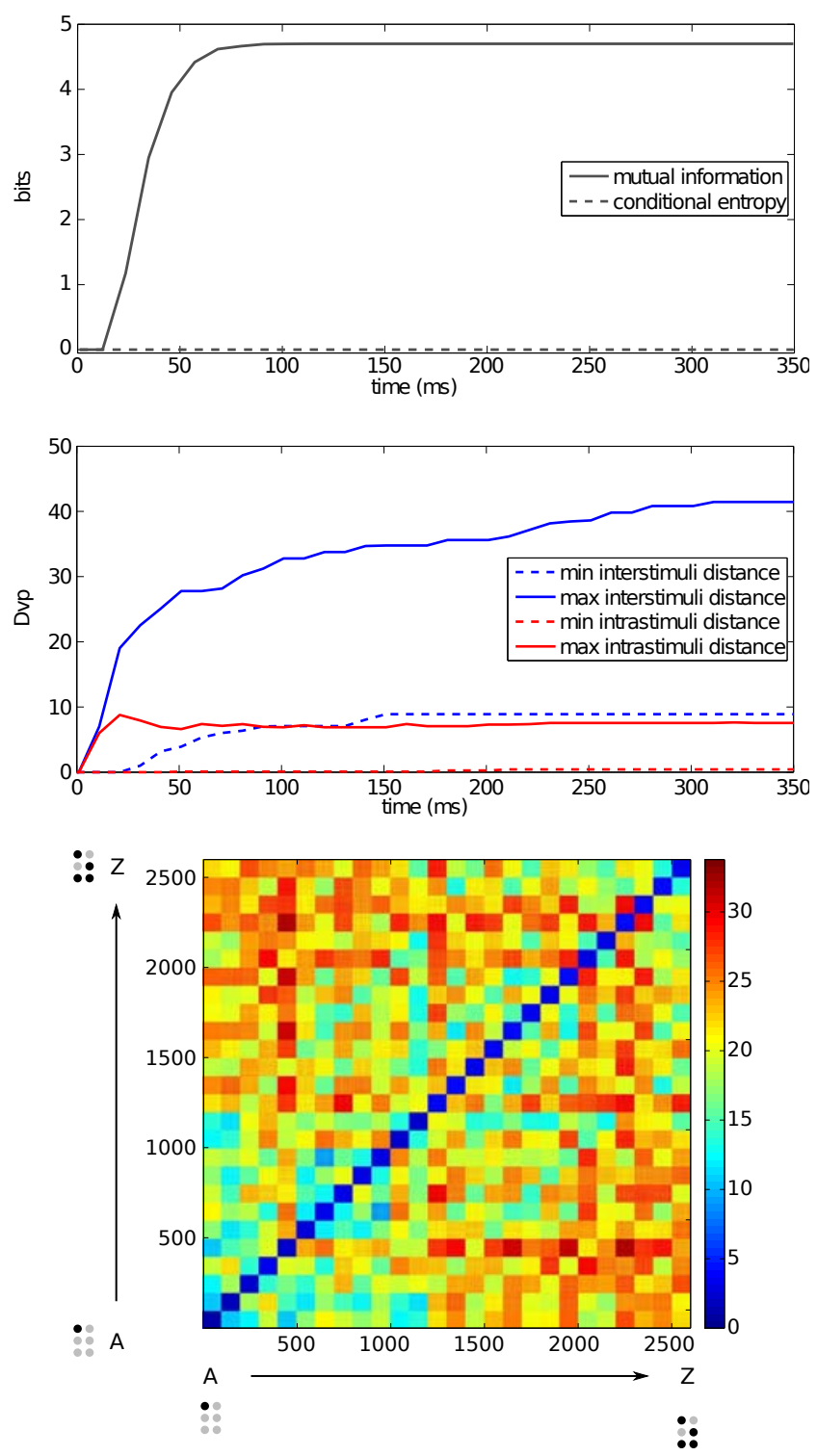

Fig. 7. Top: Metrical Mutual Information (i.e. $M I^{*}$ ) and Conditional Entropy (i.e. $H^{*}(R \mid S)$ ) are reported over time. The chosen Victor-Purpura $\operatorname{cost} C_{V P} 0.085$. The optimal discrimination criterion, namely maximum information and minimum conditional entropy, occurs after $120 \mathrm{~ms}$ from the stimulus onset. Center: Intrastimuli and interstimuli distances evolve in time as a higher number of spikes are recruited for the analsys. A perfect discrimination occurs at $120 \mathrm{~ms}$ when the minimum interstimuli distance has becomes bigger than the maximum intrastimuli one. Bottom: The distance matrix for all stimuli responses is reported. Low diagonal values can be observed corresponding to small differences between responses to the same stimulus. At the same time, the higher is the difference between the delivered stimulation patterns, the bigger are the distances between the responses.

the consequent derivation of the $D_{\text {critic }}$ leads to a perfect discrimination of the entire dynamical stimuli set.

\section{CONCLUSIONS}

A framework for the encoding/decoding process of artificial tactile signals is presented. The main result of the presented study is the fast and optimal discrimination of Braille-like stimulation patterns delivered to an artificial touch sensor. With the aim of mimicking the skin mechanore- 

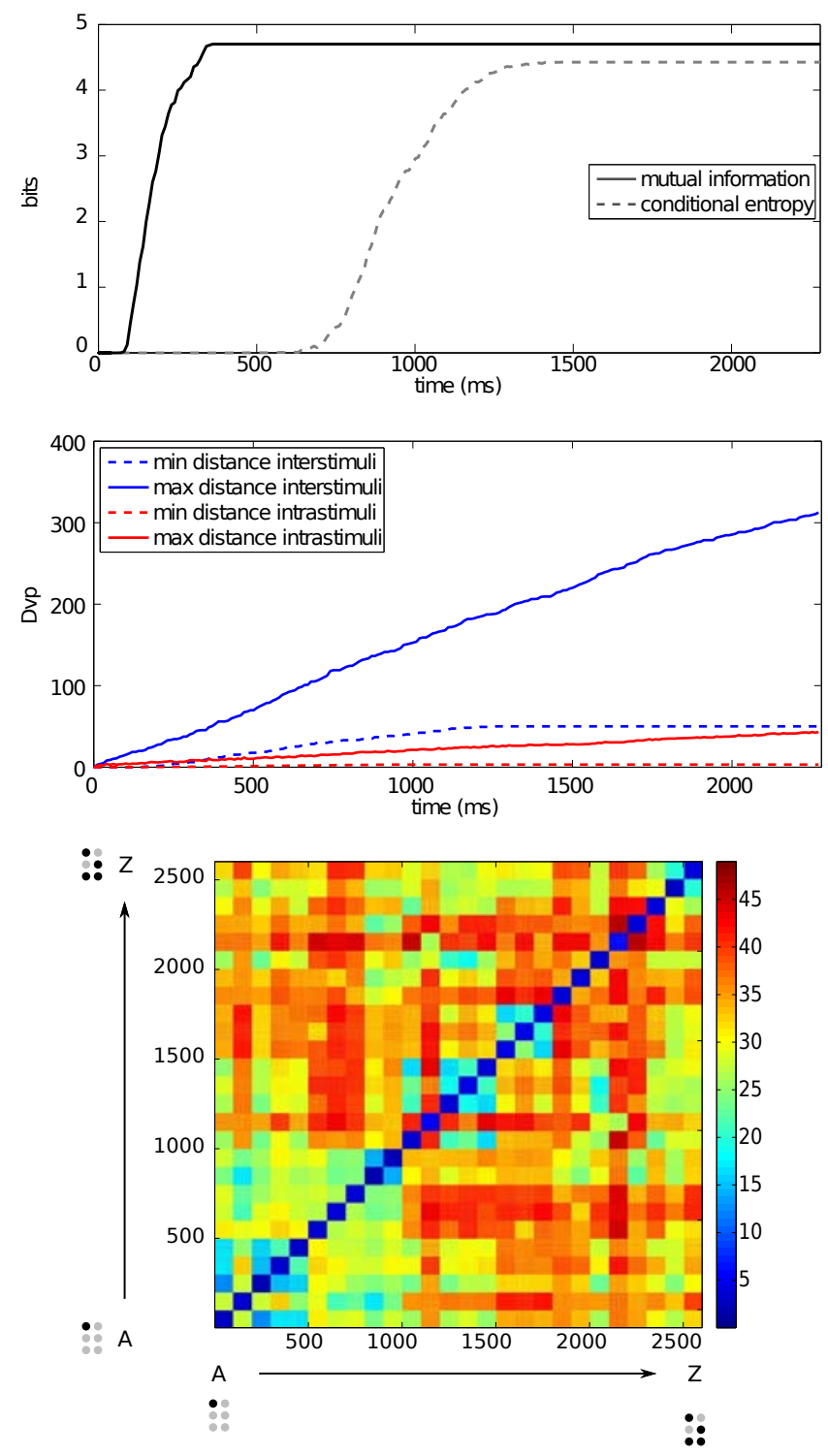

Fig. 8. Top: Metrical Mutual Information (i.e. $M I^{*}$ ) and Conditional Entropy (i.e. $H^{*}(R \mid S)$ ) are reported over time. The chosen Victor-Purpura cost $C_{V P}$ was 0.085 . As for the static stimulation, the optimal discrimination criterion (i.e. maximum information and minimum conditional entropy) is reached after $500 \mathrm{~ms}$. Center: The minimum interstimuli distance overcomes the maximum intrastimuli one at $500 \mathrm{~ms}$. Bottom: The distance matrix for all stimuli responses is reported. As already observed for the static stimulation protocol, small distances are typical of responses to the same stimulus (diagonal values) while different stimuli induce higher distances in the spike train space.

ceptor dynamics, the analogue data from the fingertip were encoded through a biologically inspired network of leaky integrate-and-fire neurons able to collect the activity of the sensors according to a specific receptive field. The encoded signals reflected the property of the tactile stimulation and were decoded on the basis of a newly defined information measure based on the metrical property of the spike train space. Such a measure allowed to perform a perfect discrimination (i.e. $100 \%$ patterns correctly classified) early after the stimulus onset (i.e. $120 \mathrm{~ms}$ for static stimuli and $500 \mathrm{~ms}$ for dynamical stimuli). Given the properties of the framework we propose and the efficacy of the encoding/decoding process, mostly interestingly with respect to the early discrimination capability, we envisage the study carried out as a possible starting point for real time robotic applications in which a fast and reliable discrmination of external sensory stimuli is required.

\section{ACKNOWLEDGMENT}

This research was granted by the EC Integrated Project SENSOPAC (SENSOrimotor structuring of Perception and Action for emerging Cognition) IST-028056-IP.

\section{REFERENCES}

[1] I. Birznieks, P. Jenmalm, A. W. Goodwin, and R. S. Johansson. Encoding of direction of fingertip forces by human tactile afferents. $J$ Neurosci, 21(20):8222-8237, October 2001.

[2] R. Brasselet, R. Johansson, and A. Arleo. Optimal context separation of spiking haptic signals by second-order somatosensory neurons. In Advances in Neural Information Processing Systems, (in press).

[3] G. Cannata, M. Maggiali, G. Metta, and G. Sandini. An embedded artificial skin for humanoid robots. In Proc. IEEE International Conference on Multisensor Fusion and Integration for Intelligent Systems MFI 2008, pages 434-438, 2008.

[4] Maurice J Chacron, Khashayar Pakdaman, and André Longtin. Interspike interval correlations, memory, adaptation, and refractoriness in a leaky integrate-and-fire model with threshold fatigue. Neural computation, 15(2):253-278, February 2003.

[5] L. Lapicque. Recherches quantitatives sur l'excitation des nerfs traité comme une polarisation. Journal de Physiologie et Pathologie General, 9:620-635, 1907.

[6] Michael London, Adi Schreibman, Michael Hausser, Matthew E. Larkum, and Idan Segev. The information efficacy of a synapse. Nat Neurosci, 5(4):332-340, April 2002.

[7] J. Phillips, R. Johansson, and K. Johnson. Representation of braille characters in human nerve fibres. Experimental Brain Research, 81(3):589-592, 1990.

[8] Rodrigo Quian Quiroga and Stefano Panzeri. Extracting information from neuronal populations: information theory and decoding approaches. Nat Rev Neurosci, 10(3):173-185, March 2009.

[9] F. Rieke, D. Warland, R. de Ruyter van Stevenick, and W. Bialek, editors. Spikes: Exploring the neural code. Cambridge: MIT Press, 1997.

[10] S. Schreiber, J. M. Fellous, D. Whitmer, P. Tiesinga, and T. J. Sejnowski. A new correlation-based measure of spike timing reliability. Neurocomputing, 52-54:925-931, June 2003.

[11] E. Shannon. A mathematical theory of communication. The Bell System Technical Journal, 27:379-423, 623-656, 1948.

[12] M C van Rossum. A novel spike distance. Neural Computation, 13(4):751-763, April 2001. PMID: 11255567.

[13] J. D. Victor and K. P. Purpura. Nature and precision of temporal coding in visual cortex: a metric-space analysis. J Neurophysiol, 76(2):13101326, August 1996. 